\title{
Gerbode Defect following Tetralogy of Fallot Repair: The Role of Transesophageal Echocardiography
}

\author{
${ }^{1}$ Suruchi Ladha, ${ }^{2}$ Uma Balasubramaniam, ${ }^{3}$ Usha Kiran, ${ }^{4}$ Neeti Makhija, ${ }^{5}$ Velayoudam Devagourou
}

\begin{abstract}
Gerbode defect is a rare type of left ventricle (LV) to right atrium (RA) shunt. It is congenital in origin, but acquired cases are also described, usually following infective endocarditis, valve replacement, trauma, or myocardial infarction. We, hereby, report two cases of acquired Gerbode defect after tetralogy of Fallot (TOF) repair, and describe the role of echocardiography in the complete visualization of the anatomical defect, and in differentiation of this shunt from other conditions. The role of transesophageal echocardiography (TEE) is highlighted during reintervention for successful shunt closure.
\end{abstract}

Keywords: Gerbode defect, Tetralogy of fallot, Transesophageal echocardiography, Tricuspid regurgitation.

How to cite this article: Ladha S, Balasubramaniam U, Kiran U, Makhija N, Devagourou V. Gerbode Defect following Tetralogy of Fallot Repair: The Role of Transesophageal Echocardiography. J Perioper Echocardiogr 2017;5(2):70-73.

\section{Source of support: Nil}

Conflict of interest: None

\section{INTRODUCTION}

Gerbode defect is a communication between the LV and the RA described by Gerbode et al. ${ }^{1}$ This shunt is usually congenital in origin, representing less than $1 \%$ of all congenital heart defects. ${ }^{2}$ However, rarely, it can be acquired after cardiac surgery. Few cases of acquired LV-to-RA shunts have been reported in the literature after cardiac surgery involving the aortic valve, mitral valve, or ventricular septal defect (VSD) repair. ${ }^{3}$ In the same clinical scenario, tricuspid regurgitation (TR) is also common in the postoperative period due to associated RV dysfunction or pulmonary hypertension. On echocardiographic examination, this jet of blood around the tricuspid valve (TV), and directed toward the RA, may not always be due to TR. ${ }^{2}$ An anesthesiologist should be

\footnotetext{
${ }^{1,2}$ Senior Resident, ${ }^{3}$ Professor and Head, ${ }^{4,5}$ Professor

${ }^{1-4}$ Department of Cardiac Anesthesia, All India Institute of Medical Sciences, New Delhi, India

${ }^{5}$ Department of Cardiothoracic and Vascular Surgery, All India Institute of Medical Sciences, New Delhi, India

Corresponding Author: Uma Balasubramaniam, Senior Resident, Department of Cardiac Anesthesia, All India Institute of Medical Sciences, New Delhi, India, Phone: +911126593423 e-mail: uma_dilli@yahoo.co.in
}

aware of the uncommon possibility of the existence of an LV-to-RA shunt, suggesting an acquired Gerbode defect, because the successful management of this condition will require a reintervention.

We report two cases of acquired Gerbode defect following intracardiac repair of TOF and its clinical challenges. We describe the role of TEE in the diagnosis of acquired Gerbode following TOF repair, and the importance of intraoperative diagnosis for better patient prognosis. The misinterpretation of Gerbode defect as a high-velocity jet of TR leads to the incorrect diagnosis of an elevated right ventricular systolic pressure. This can be prevented by the awareness and suspicion of such a rare defect.

\section{CASE REPORTS}

\section{Case 1}

A 5-year-old boy, with diagnosis of TOF with a single subaortic VSD, underwent intracardiac repair with infundibular resection and transannular patch. The patient was weaned from cardiopulmonary bypass (CPB) on high inotropic support. The TEE detected mild TR and RV dysfunction. There was no residual VSD. During the postoperative period, patient had low cardiac output with persistent respiratory distress. He had shortness of breath and decreased functional capacity. On auscultation, there was a $4 / 6$ holosystolic murmur, heard maximal at the left third intercostal space. Signs of right heart failure with massive hepatomegaly, bilateral pleural effusion, elevated jugular venous pressure, peripheral edema, and bilateral pulmonary rales were present during the 1st week of postoperative recovery. Chest X-ray anteroposterior view showed evidence of pulmonary congestion. The electrocardiogram showed tall, peaked right atrial $\mathrm{P}$ waves. The clinical evidence suggested RV dysfunction following TOF repair; however, postoperative transthoracic echocardiogram revealed an LV-to-RA shunt with peak velocity of $3.4 \mathrm{~m} / \mathrm{s}$, significant RA dilatation, and right-ventricular dilatation with dysfunction. A redo surgery for closure of the shunt was planned. Intraoperative TEE using S7-3t Philips ultrasound probe and ultrasound machine (Phillips iE33 model, Bothell, Washington, USA) confirmed the presence of an abnormal systolic jet entering the RA from the LV, above the TV (Fig. 1). The TEE imaging 


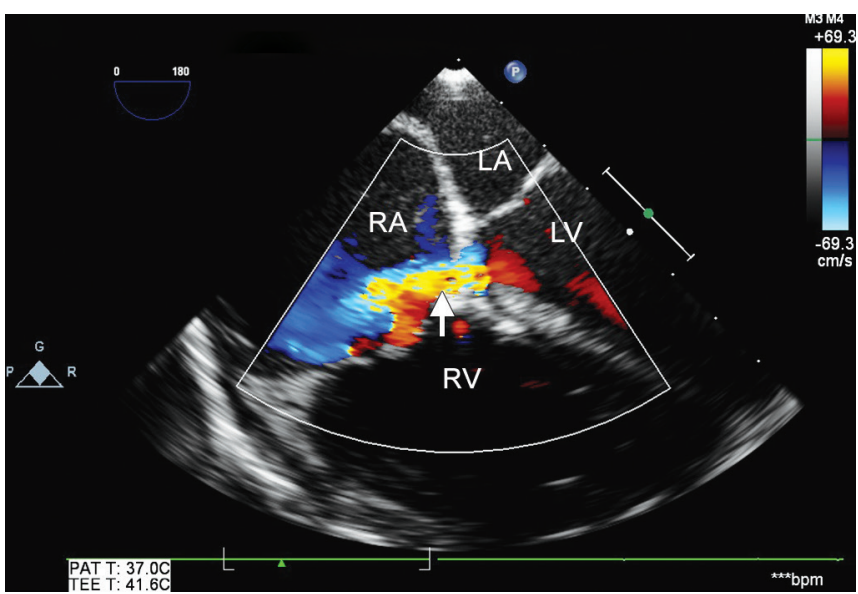

Fig. 1: Two-dimensional midesophageal four-chamber view at $0^{\circ}$ angle with color flow Doppler showing a jet entering RA through TV annulus, bowing of interatrial septum to left, and an enlarged RV

was performed in multiple planes to evaluate the origin of the shunt jet. The color Doppler imaging showed a jet originating from the LV and entering into the RA (Video 1). The opening of the VSD appeared to be small and well defined. Continuous wave Doppler showed high jet velocity with peak gradient of $48 \mathrm{~mm} \mathrm{Hg}$ (Fig. 2).

On surgical exposure, the opening of the VSD was seen on the atrial aspect of the hinge of the TV. The surgeon insufflated the RV with cold saline; the TV was found to be competent. No saline passed through the defect indicating that the acquired defect was directly between the LV and the RA. The VSD was closed with an autologous pericardial patch. The patient had an uneventful postoperative recovery.

\section{Case 2}

The second case was a 6-year-old female patient of TOF scheduled for intracardiac repair, with transannular

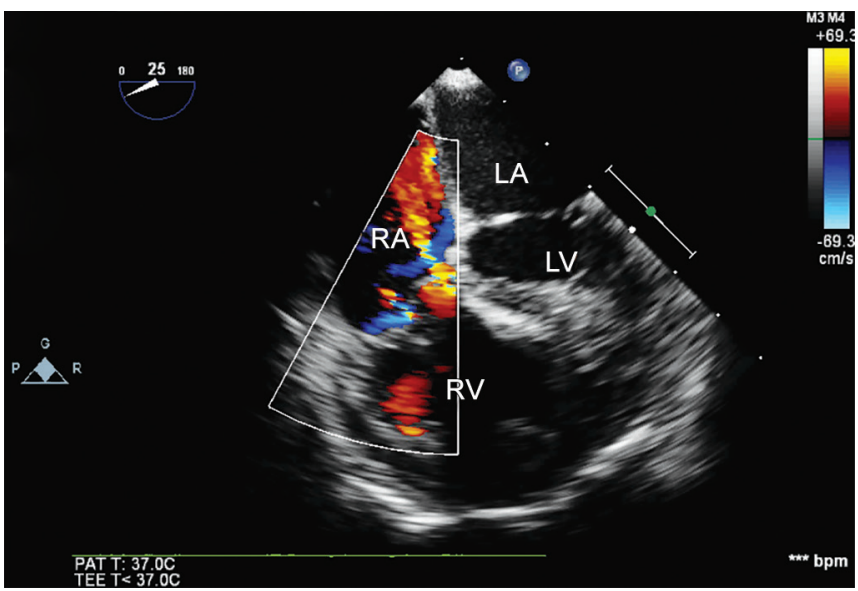

Fig. 3: Two-dimensional midesophageal four-chamber view at an angle of $25^{\circ}$ with color flow Doppler showing a jet from the LV to RA

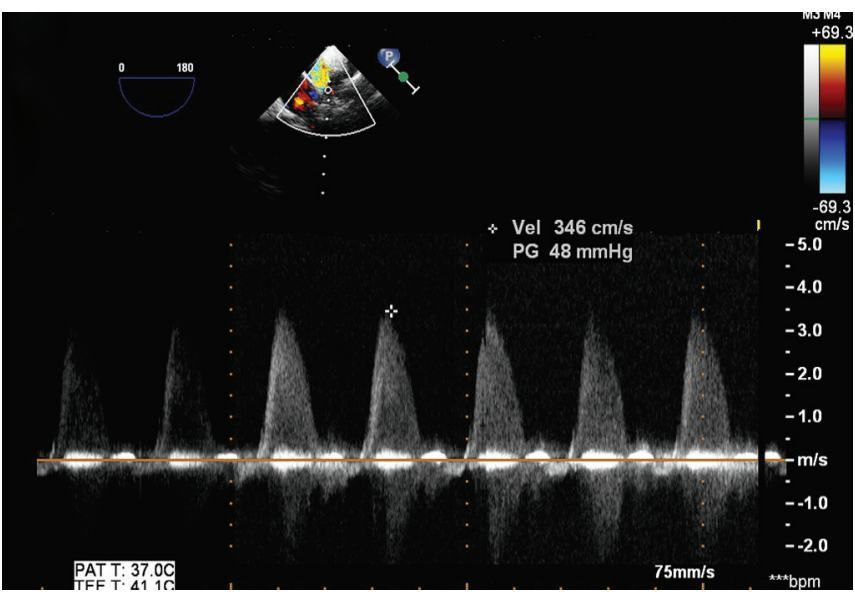

Fig. 2: Two-dimensional midesophageal four-chamber view at $0^{\circ}$ angle with continuous wave Doppler revealing a high-velocity systolic jet toward RA (maximum velocity of $3.4 \mathrm{~m} / \mathrm{s}$ ) and maximum peak gradient of $48 \mathrm{~mm} \mathrm{Hg}$

patch. Weaning from $\mathrm{CPB}$ could not be accomplished as the patient had severe RV dysfunction with a dilated RV. Intraoperative TEE using S7-3t Philips ultrasound probe and ultrasound machine (Phillips iE33 model, Bothell, Washington, USA) revealed an abnormal systolic jet entering the RA from the LV (Fig. 3, Video 2). The TR was ruled out after performing TEE examinations in multiple views and planes. The peak gradient on continuous wave Doppler examination of the jet was $52 \mathrm{~mm} \mathrm{Hg}$ with a jet velocity of $3.2 \mathrm{~m} / \mathrm{s}$ (Fig. 4). Such high values were consistent with a Gerbode defect. The defect was closed, and patient was weaned successfully from CPB. The patient had an uneventful recovery and postoperative TEE images showed no residual VSD.

\section{DISCUSSION}

Left ventricular-to-right atrial (LV-RA) communications are rare intracardiac defects resulting from ventricular

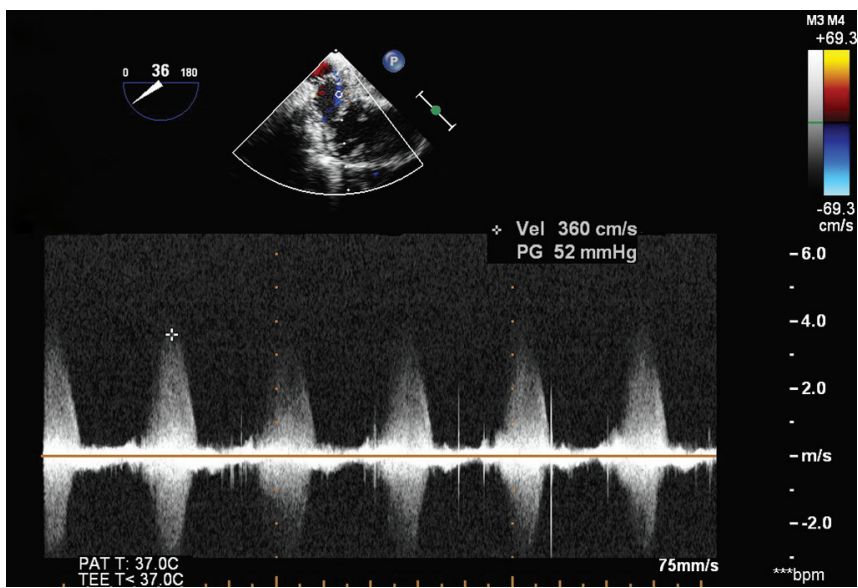

Fig. 4: Two-dimensional midesophageal four-chamber view at $36^{\circ}$ angle with continuous wave Doppler revealing a high-velocity systolic jet toward RA (maximum velocity of $3.6 \mathrm{~m} / \mathrm{s}$ ) and maximum peak gradient of $52 \mathrm{~mm} \mathrm{Hg}$ 
septal perforation due to congenital or acquired causes. These defects are collectively known as Gerbode defects. ${ }^{2}$ Both congenital and acquired forms result from a defect in the membranous interventricular septum. The TV is located apically in comparison with the mitral valve. A thin part of the septum between them may be ruptured during cardiac surgery and create a direct shunt from LV into RA. ${ }^{4}$

There are two types of defects known, a direct and an indirect as described by Gerbode et al. ${ }^{1}$

In type I (indirect type), the shunt is from LV to RV through the perimembranous VSD and then through the TV into the RA. The communication thus occurs below the TV. In type II (direct type), blood in the LV flows through the small area of membranous septum into the RA. This communication is above the TV and is rarer than the indirect variant. Both our cases had abnormal systolic jet entering the RA from the LV, thereby, suggesting direct type of Gerbode defect.

When classifying it anatomically, the Gerbode defect may be above the TV, below the TV, or a combination of these two. ${ }^{4}$ If its relation is below the TV, this defect accompanies a second defect located in the TV, usually in its septal leaflet. Because of the rarity of this defect and its close association with the TV, it may be misinterpreted as a TR jet on echocardiography. ${ }^{2,5}$ The TR must be diagnosed promptly because of poor tolerance of postoperative RV insufficiency. The TR occurs as a consequence of TV distortion following VSD closure or as a sequence of right heart failure or pulmonary hypertension.

The TEE is helpful in distinguishing this defect from conditions like TR, ruptured sinus of Valsalva (RSOV) aneurysms, or aortoatrial shunts. In the acquired Gerbode defect, the communication is usually above the insertion point of the TV leaflet. The locus of this high systolic flow from the membranous septum helps in differentiation from TR as seen in our case. Differences in the timing of the shunt flow can help in differentiating it from the RSOV and aortoatrial shunt. During systole, Gerbode defect typically produces left-to-right shunt as in our cases, while RSOV aneurysms will produce diastolic shunting resulting from the diastolic gradient between the aorta and RA. The aortoatrial shunts, on the contrary, have continuous flow across the shunt both during systole and diastole. ${ }^{6}$ A Gerbode defect should be suspected when echocardiographic interrogation reveals an unusually dilated RA and abnormal bulging of the interatrial septum to the left side as seen in our case. Color flow Doppler is valuable in revealing high-velocity systolic flow ( $>4 \mathrm{~m} / \mathrm{s}$ ) originating from the upper membranous septum and directed toward the RA. In our case, velocity was, however, relatively low due to the low cardiac output state post intracardiac repair. The other diagnostic feature is the high Doppler gradient between the LV and RA, as in our patients the peak gradient was above $48 \mathrm{~mm} \mathrm{Hg}{ }^{7}$ The gradient reflects the notable difference between the LV systolic pressure and the low RA pressure. Color Doppler can be useful for delineating the origin and direction of the different jets. Yan et al have reported that continuous wave and pulse wave Doppler imaging of an LV-RA shunt are often misinterpreted as TR. ${ }^{8}$ In both Gerbode defect and TR, the imaging of the inferior vena cava and hepatic veins may show dilated veins without respiratory variability. Silbiqer et $\mathrm{al}^{4}$ specified a few other echocardiographic features suggestive of a Gerbode defect, like atypical jet direction, persistent shunt flow in diastole, and normal diastolic pulmonary artery pressure as estimated from pulmonary regurgitation jet. The other differential diagnoses of Gerbode defect include VSD and RSOV.

Some authors have reported that transthoracic echocardiography may be more sensitive for the diagnosis as these jets are anteriorly directed, toward the RA, whereas others have reported that it may be missed on transthoracic echocardiography due to acoustic shadowing and eccentricity of the jet. ${ }^{8,9}$ However, in general, the diagnostic accuracy of TEE is considered to be higher. ${ }^{8}$

Perioperative diagnosis of Gerbode defect following TOF is often difficult. These two cases did not have high gradients required for diagnosis of Gerbode defect, but it may not be true in every case. Relying on gradients for diagnosis might not be possible in the early postoperative period or low cardiac output states. In our first case, the suspicion of Gerbode defect was raised through clinical and echocardiographic correlations. The LV-to-RA shunting causes most of these patients to have significant symptoms. The prominent physical finding of an LV-toRA shunt is a loud and harsh pansystolic murmur, often associated with a thrill along the left sternal border, which may also occur with a residual VSD. Nevertheless, a diagnosis is usually not possible based on the physical examination alone and requires confirmation with echocardiographic examination.

Spontaneous closure of these defects is very rare and surgical repair is excellent, but technically challenging. ${ }^{7}$ In small defects, direct closure is possible, but larger ones require a patch of pericardium. As an alternative to surgery, there are reported cases of percutaneous closure with septal occluders. ${ }^{10}$ In our case, surgical closure was performed. Re-exploration is always a risk, and the correction of a shunt may be very demanding even with an experienced surgeon. 


\section{CONCLUSION}

To conclude, acquired LV-to-RA defect is a rare type of VSD. The diagnosis of LV-to-RA communication is not always easy. There should be a high index of suspicion of Gerbode defect in a patient not normally recovering after intracardiac repair and in a patient of TOF. The detection of a Gerbode defect is a diagnostic challenge and a potential source of misinterpretation. A focused and detailed TEE has a central role in both diagnosis and management.

\section{REFERENCES}

1. Gerbode F, Hultgren H, Melrose D, Osborn J. Syndrome of left ventricular-right atrial shunt; successful surgical repair of defect in five cases, with observation of bradycardia on closure. Ann Surg 1958 Sep;148(3):433-446.

2. Hansalia S, Manda J, Pothineni KR, Nanda NC. Usefulness of live/real time three-dimensional transthoracic echocardiography in diagnosing acquired left ventricular-right atrial communication misdiagnosed as severe pulmonary hypertension by two-dimensional transthoracic echocardiography. Echocardiography 2009 Feb;26(2):224-227.

3. Demirkol S, Gurkan Yesil F, Bozlar U, Balta S, Sahin MA, Guler A. Multimodality imaging of a congenital Gerbode defect. Kardiol Pol 2013 Jan;71(1):104.
4. Silbiqer JJ, Kamran, M, Handwerker, S, Kumar N, Marcali M. The Gerbode defect: left ventricular to right atrial communication-anatomic, hemodynamic, and echocardiographic features. Echocardiography 2009 Sep;26(8):993-998.

5. Xhabija N, Prifti E, Allajbeu I, Sula F. Gerbode defect following endocarditis and misinterpreted as severe pulmonary arterial hypertension. Cardiovasc Ultrasound 2010 Sep;8:44.

6. Ananthasubramaniam K. Clinical and echocardiographic features of aorto-atrial fistulas. Cardiovasc Ultrasound 2005 Jan;3(1):1-5.

7. Matt $\mathrm{P}$, Winkler B, Carrel T, Eckstein F. Plicated patch repair for acquired Gerbode defect involving the tricuspid valve. Ann Thorac Surg 2010 Feb;89(2):643-645.

8. Yuan SM. A systematic review of acquired left ventricle to right atrium shunts (Gerbode defects). Hellenic J Cardiol 2015 Mar;56(5):357-372.

9. Ota T, Yamaguchi R, Taniqawa T, Otuka K, Hayashi Y, Nishiyama $H$. Left ventricular-right atrial communication by perforation of the atrioventricular portion of the membranous septum and severe valve regurgitation caused by infective endocarditis. J Echocardiogr 2011 Mar;9(1):30-32.

10. Fanari Z, Barekatain A, Abraham N, Hopkins JT. Percutaneous closure of acquired Gerbode defect: management of a rare complication of cardiac surgery. Interact Cardiovasc Thorac Surg 2015 Jul;21(1):127-128. 